Case Report

\title{
The Closed-Loop System Improved the Control of a Pregnant Patient with Type 1 Diabetes Mellitus
}

\author{
Guillermo E. Guzmán Gómez ${ }^{(\mathbb{D}},{ }^{1}$ Julian A. Viggiano, ${ }^{2}$ A. Silva-De Las Salas, ${ }^{3}$ \\ Veline Martínez, ${ }^{4}$ and María A. Urbano Bonilla ${ }^{5}$ \\ ${ }^{1}$ Fundación Clínica Valle del Lili, Department of Endocrinology, Universidad Icesi, Cali, Colombia \\ ${ }^{2}$ PGY3 Internal Medicine Resident, Fundación Clínica Valle del Lili, Universidad Icesi, Cali, Colombia \\ ${ }^{3}$ Universidad Icesi, Cali, Colombia \\ ${ }^{4}$ Fundación Clínica Valle del Lili, Department of Internal Medicine, Universidad Icesi, Cali, Colombia \\ ${ }^{5}$ Fundación Clínica Valle del Lili, Clinical Investigations Center, Cali, Colombia \\ Correspondence should be addressed to Guillermo E. Guzmán Gómez; guillermoeguzman@gmail.com
}

Received 19 May 2021; Accepted 11 September 2021; Published 21 September 2021

Academic Editor: Osamu Isozaki

Copyright (C) 2021 Guillermo E. Guzmán Gómez et al. This is an open access article distributed under the Creative Commons Attribution License, which permits unrestricted use, distribution, and reproduction in any medium, provided the original work is properly cited.

\begin{abstract}
Objective. Closed-loop insulin systems represent a technological advance in diabetes management but have rarely been studied in pregnancy. We report a case of a patient with type 1 diabetes mellitus who was previously a user of the Paradigm VEO pump and then migrated to Medtronic 670G. Research Design and Methods. We reviewed the case of a G1P0 patient with type 1 diabetes, treated with the Medtronic 670G system during pregnancy; a comparison with current literature was done. Results. The patient achieved improved glycemic control as measured by time spent in different ranges as follows: $<70 \mathrm{mg} / \mathrm{dL}, 8-4 \%$ and $70-180 \mathrm{mg} /$ $\mathrm{dL}, 83-94 \%$. Secondary outcomes included reduction of stress, anxiety, fear of hypoglycemia, and the psychological burden related to the disease. There were no obstetric or neonatal complications. Conclusion. The Medtronic 670G closed-loop system was used safely in a pregnant woman; nevertheless, further research is needed to validate this system in this patient population.
\end{abstract}

\section{Introduction}

Type 1 diabetes mellitus (DM1) increases the risk of obstetric and neonatal complications [1]. During gestation, hormonal changes cause a diabetogenic physiological state which can lead to volatile glycemic control in diabetic patients [2]. Previous studies have shown that DM1 women are up to five times more likely to experience severe hypoglycemia during early pregnancy compared to the preconception period [3]; the management of these patients can be challenging, and there is a need for new therapies. Advances in biotechnology seek to achieve glycemic control and minimize the burden of hypoglycemia.

The Medtronic MiniMed 670G is a closed-loop hybrid insulin infusion system approved for patients with diabetes $[4,5]$; however, safety in pregnancy has not been established through clinical trials. One of the challenges with this pump is that its algorithm targets fasting glucose of $120 \mathrm{mg} / \mathrm{dL}$, higher than the standard target of $<95 \mathrm{mg} / \mathrm{dL}$ during pregnancy [6-10]. Herein, we present the case of a female patient with DM1 who achieved satisfactory glycemic control using the MiniMed 670G pump.

\section{Methods}

A 33-year-old woman with a history of DM1 since 16 years, user of an insulin pump (Paradigm ${ }^{\circledR}$ Veo system) due to high glycemic variability and recurrent hypoglycemia, presented to the outpatient clinic seeking preconception counseling. She reported no hypoglycemic episodes since 2015 and no macrovascular or microvascular complications.

She subsequently reported her pregnancy status at week 10. At the beginning of pregnancy, her weight was $57 \mathrm{Kg}$, 
TABLE 1: Glycemic control monitoring.

\begin{tabular}{|c|c|c|c|c|c|c|c|c|}
\hline \multirow{2}{*}{ Follow-up week } & \multirow{2}{*}{ Pump } & \multirow{2}{*}{ Mode } & \multicolumn{4}{|c|}{ Time in range (TIR) } & \multirow{2}{*}{ TDD (UI) } & \multirow{2}{*}{ Basal/bolus ratio (\%) } \\
\hline & & & $<54 \mathrm{mg} / \mathrm{dl}(\%)$ & $54-63 \mathrm{mg} / \mathrm{dl}(\%)$ & $63-140 \mathrm{mg} / \mathrm{dl}(\%)$ & $>140 \mathrm{mg} / \mathrm{dl}$ & & \\
\hline $30^{*}$ & VEO & N/A & 1 & 4 & 87 & 8 & 40 & $38 / 62$ \\
\hline $32^{*}$ & $670 \mathrm{G}$ & Manual & 1 & 2 & 80 & 17 & 45 & $33 / 67$ \\
\hline $34^{*}$ & $670 \mathrm{G}$ & Automatic & 0 & 1 & 84 & 15 & 46 & $28 / 72$ \\
\hline $38^{*}$ & $670 \mathrm{G}$ & Automatic & 0 & 2 & 96 & 2 & 34 & $24 / 76$ \\
\hline $1^{* *}$ & $670 \mathrm{G}$ & Manual & 1 & 2 & 94 & 3 & 29 & $21 / 79$ \\
\hline $4^{* *}$ & $670 \mathrm{G}$ & Manual & 0 & 1 & 92 & 7 & 22 & $45 / 55$ \\
\hline $6^{* *}$ & $670 \mathrm{G}$ & Automatic & 0 & 1 & 87 & 12 & 23 & $35 / 66$ \\
\hline
\end{tabular}

*Follow-up during pregnancy. ${ }^{* *}$ Postpartum follow-up. TDD, total daily dose.

body mass index (BMI) $23 \mathrm{Kg} / \mathrm{m}^{2}$, and glycated hemoglobin (HbA1c) 6.7\%. At week 24, laboratories showed HbAlc of $6.3 \%$; however, she developed high glycemic variability and inadvertent hypoglycemias. Enlite Sensor ${ }^{\circledR}$ continuous glucose monitoring (CGM) dysfunction was discovered, and a switch to a MiniMed 670G pump technology was decided.

During the following four weeks, the Paradigm ${ }^{\circledR}$ Veo pump was kept without the use of a sensor, precipitating hypoglycemia with self-reported anxiety and fear of a drop in glucose levels. At week 28, maternal weight was $63 \mathrm{Kg}$, and the estimated fetal weight was $1230 \mathrm{~g}$ for a 67 th percentile (P67). At week 30, the training process on MiniMed $670 \mathrm{G}$ began, a protocol that started with two weeks in the manual mode and was then switched to the automatic mode as an off-label indication. This optimized the times in range and reduced the hypoglycemic episodes and hence the related anxiety in the patient.

By week 33, the estimated fetal weight was $2291 \mathrm{~g}$ (P88) and at week 35 was $2832 \mathrm{~g}$ (P95) with a maternal weight of $65 \mathrm{Kg}$. The glycemic control ranges remained within goal until the end of the pregnancy, which took place at week 38 via cesarean section, during which the automatic mode and dextrose infusion were maintained (Table 1). The glycemic variability was higher in weeks 30-34 with higher time in range in hyperglycemia and hypoglycemia.

There were no obstetric complications, and a healthy female newborn weighting $3635 \mathrm{~g}$, suitable for gestational age (P80), was born. In the immediate postpartum period, the automatic mode was suspended due to recurrent hypoglycemic episodes and the periodic suspension of the system by the patient. The manual mode was continued for four weeks, requiring weekly adjustments due to hypoglycemia and a significant decrease in the total required daily insulin dose. At the end of the fourth postpartum week, the automatic mode was restarted, and good glycemic control was maintained.

\section{Discussion}

DM1 increases the risk of obstetric and neonatal complications associated with maternal hyperglycemia in pregnancy such as macrosomia, congenital anomalies, shoulder dystocia, stillbirth, and neonatal death $[1,2,11-13]$. The glucose goals of pregnant patients are stricter: fasting and preprandial target glucose is $<90 \mathrm{mg} / \mathrm{dL}$. Therefore, various pharmacological management options have been developed with less variable insulin regimens that promote better glycemic control in pregnant women [14]. The insulin infusion pump (IIP) is a treatment strategy used when insulin regimens have not been able to achieve adequate control. This syste $m$ has proven to be effective in reducing $\mathrm{HbAlc}$ in the nonpregnant population [15]. Along with the development of IIP systems, continuous glucose monitoring has emerged, and in combination, they improve clinical outcomes related to glycemic control. In pregnancy, the use of an insulin pump over multiple-dose injection therapy has not shown greater benefit [16-19]. However, the CONCEPTT trial demonstrated that the use of CGM helps improve neonatal outcomes and reduce maternal hyperglycemia [20].

Research has focused on the development of an artificial pancreas, which, through prediction algorithms, can increase or decrease the supply of insulin according to glucose levels obtained with CGM [21-24]. Stewart et al. compared a closed-loop system from a DANA Diabecare R insulin pump (SOOIL) and a CGM supported by FreeStyle Navigator II vs. an open-loop system in DM1 patients. Their findings reported that women under the closed-loop system achieved better glycemic control, evidenced by fewer obstetric and neonatal complications [25].

In 2016, the FDA approved the first hybrid system, the MiniMed 670G. This system differs from others since it has an automatic glycemic control algorithm that targets a nonmodifiable glucose goal of $120 \mathrm{mg} / \mathrm{dL}$ which is higher than the standard target for pregnant patients. The TIR International Consensus established that the target range for pregnant patients is $63-140 \mathrm{mg} / \mathrm{dL}$ and to stay below this range less than 5\% of the time and above of it less than $25 \%$ of the time [26].

Given the short time, this system has been on the market, and there are few reports of its use during gestation. In the case of our patient, migrating from the VEO system to $670 \mathrm{G}$ reduced hypoglycemia and increased time in the target glucose range at rates higher than what is reported in literature [27].

Additionally, the control of stress, anxiety, fear of hypoglycemia, and the psychological burden of the disease play an important role in the management of the diabetic patient. The use of CGM in the CONCEPTT trial showed a reduction in anxiety related to fear of hypoglycemia [20], improving the quality of life of patients using IIP systems coupled with CGM [28]. 


\section{Conclusion}

We report an adequate glycemic control in a pregnant patient with DM1 who was transitioned to a closed-loop system at 30 weeks of gestation. The patient had no macrovascular or microvascular complications. Patient showed decreased episodes of severe hypoglycemia and hyperglycemia with better glycemic control than previously reported cases. Our findings are encouraging and open the door for further clinical studies on the use of closed-loop systems in pregnancy.

\section{Consent}

The patient has provided consent to the publication of this case.

\section{Conflicts of Interest}

The authors declare that they have no conflicts of interest.

\section{References}

[1] J. P. Vandorsten, W. C. Dodson, M. A. Espeland et al., "NIH consensus development conference: diagnosing gestational diabetes mellitus," NIH Cconsensus and Sate-of-the-Science Statements, vol. 29, no. 1, pp. 1-31, 2013.

[2] T. A. Buchanan and A. H. Xiang, "Gestational diabetes mellitus," Journal of Clinical Investigation, vol. 115, no. 3, pp. 485-491, 2005.

[3] L. R. Nielsen, U. Pedersen-Bjergaard, B. Thorsteinsson, M. Johansen, P. Damm, and E. R. Mathiesen, "Hypoglycemia in pregnant women with type 1 diabetes: predictors and role of metabolic control," Diabetes Care, vol. 31, no. 1, pp. 9-14, 2008.

[4] S. K. Garg, S. A. Weinzimer, W. V. Tamborlane et al., "Glucose outcomes with the in-home use of a hybrid closed-loop insulin delivery system in adolescents and adults with type 1 diabetes," Diabetes Technology \& Therapeutics, vol. 19, no. 3, pp. 155-163, 2017.

[5] M. de Bock, J. Dart, A. Roy et al., "Exploration of the performance of a hybrid closed loop insulin delivery algorithm that includes insulin delivery limits designed to protect against hypoglycemia," Journal of Diabetes Science and Technology, vol. 11, no. 1, pp. 68-73, 2016.

[6] J. L. Ruiz, J. L. Sherr, E. Cengiz et al., "Effect of insulin feedback on closed-loop glucose control: a crossover study," Journal of Diabetes Science and Technology, vol. 6, no. 5, pp. 1123-1130, 2012.

[7] G. M. Steil, "Algorithms for a closed-loop artificial pancreas: the case for proportional-integral-derivative control," Journal of Diabetes Science and Technology, vol. 7, no. 6, pp. 16211631, 2013.

[8] S. A. Weinzimer, G. M. Steil, K. L. Swan, J. Dziura, N. Kurtz, and W. V. Tamborlane, "Fully automated closed-loop insulin delivery versus semiautomated hybrid control in pediatric patients with type 1 diabetes using an artificial pancreas," Diabetes Care, vol. 31, no. 5, pp. 934-939, 2008.

[9] Panel, IA of D and PSGC, International association of diabetes and pregnancy study groups recommendations on the diagnosis and classification of hyperglycemia in pregnancy," Diabetes Care, vol. 33, no. 3, pp. 676-682, 2010.
[10] A. D. Association, "Management of diabetes in pregnancy: standards of medical care in diabetes-2020," Diabetes Care, vol. 43, no. Supplement 1, pp. S183-S192, 2020.

[11] S. Handwerger and M. Freemark, "The roles of placental growth hormone and placental lactogen in the regulation of human fetal growth and development," Journal of Pediatric Endocrinology and Metabolism, vol. 13, no. 4, pp. 343-356, 2000.

[12] L. Barbour, "New concepts in insulin resistance of pregnancy and gestational diabetes: long-term implications for mother and offspring," Journal of Obstetrics and Gynaecology, vol. 23, no. 5, pp. 545-549, 2003.

[13] H. Yamashita, J. Shao, and J. E. Friedman, "Physiologic and molecular alterations in carbohydrate metabolism during pregnancy and gestational diabetes mellitus," Clinical Obstetrics and Gynecology, vol. 43, no. 1, pp. 87-98, 2000.

[14] L. R. Mack and P. G. Tomich, "Gestational diabetes: diagnosis, classification, and clinical care gestational diabetes," Obstetrics and Gynecology Clinics of North America, vol. 44, no. 2, pp. 207-217, 2017.

[15] G. Guzmán, V. Martínez, J. D. Yara et al., "Glycemic control and hypoglycemia in patients treated with insulin pump therapy: an observational study," Journal of Diabetes Research, vol. 2020, Article ID 1581726, 2020.

[16] O. Cohen, N. Keidar, M. Simchen, B. Weisz, M. Dolitsky, and E. Sivan, "Macrosomia in well controlled CSII treated type I diabetic pregnancy," Gynecological Endocrinology, vol. 24, no. 11, pp. 611-613, 2008.

[17] D. Bruttomesso, M. Bonomo, S. Costa et al., "Type 1 diabetes control and pregnancy outcomes in women treated with continuous subcutaneous insulin infusion (CSII) or with insulin glargine and multiple daily injections of rapid-acting insulin analogues (glargine-MDI)," Diabetes and Metabolism, vol. 37, no. 5, pp. 426-431, 2011.

[18] D. Farrar, D. J. Tuffnell, J. West, and H. M. West, "Continuous subcutaneous insulin infusion versus multiple daily injections of insulin for pregnant women with diabetes," Cochrane Database of Systematic Reviews, vol. 6, Article ID CD005542, 2016.

[19] G. Petrovski, C. Dimitrovski, M. Bogoev, T. Milenkovic, I. Ahmeti, and I. Bitovska, "Is there a difference in pregnancy and glycemic outcome in patients with type 1 diabetes on insulin pump with constant or intermittent glucose monitoring? A pilot study," Diabetes Technology \& Therapeutics, vol. 13, no. 11, pp. 1109-1113, 2011.

[20] D. S. Feig, L. E. Donovan, R. Corcoy et al., "Continuous glucose monitoring in pregnant women with type 1 diabetes (CONCEPTT): a multicentre international randomised controlled trial," Lancet (London, England), vol. 390, no. 10110, pp. 2347-2359, 2017.

[21] C. A. Combs, "Continuous glucose monitoring and insulin pump therapy for diabetes in pregnancy," Journal of $M a$ ternal-Fetal and Neonatal Medicine, vol. 25, no. 10, pp. 2025-2027, 2012.

[22] J. Kropff and J. H. DeVries, "Continuous glucose monitoring, future products, and update on worldwide artificial pancreas projects," Diabetes Technology \& Therapeutics, vol. 18, no. Suppl 2, pp. S253-S263, 2016.

[23] M. Breton, A. Farret, D. Bruttomesso et al., "Fully integrated artificial pancreas in type 1 diabetes: modular closed-loop glucose control maintains near normoglycemia," Diabetes, vol. 61, no. 9, pp. 2230-2237, 2012.

[24] R. Hovorka, K. Kumareswaran, J. Harris et al., "Overnight closed loop insulin delivery (artificial pancreas) in adults with 
type 1 diabetes: crossover randomised controlled studies," $B M J$, vol. 342, 2011.

[25] Z. A. Stewart, M. E. Wilinska, S. Hartnell et al., "Closed-loop insulin delivery during pregnancy in women with type 1 diabetes," New England Journal of Medicine, vol. 375, no. 7, pp. 644-654, 2016.

[26] M. Kalligeros, F. Shehadeh, E. K. Mylona et al., "Association of obesity with disease severity among patients with coronavirus disease 2019," Obesity (Silver Spring), vol. 28, no. 7, pp. 1200-1204, 2020.

[27] S. Polsky and H. K. Akturk, "Case series of a hybrid closedloop system used in pregnancies in clinical practice," Diabetes/Metabolism Research and Reviews, vol. 36, no. 3, Article ID e3248, 2020.

[28] H. Ghazanfar, S. Rizvi, A. Khurram, F. Orooj, and I. Qaiser, "Impact of insulin pump on quality of life of diabetic patients," Indian Journal of Endocrinology and Metabolism, vol. 20, no. 4, p. 506, 2016. 